\title{
Electroanalysis
}

\section{Diamond electrodes for high sensitivity mercury detection in the aquatic environment: Influence of surface preparation and gold nanoparticle activity \\ --Manuscript Draft--}

\begin{tabular}{|c|c|}
\hline Manuscript Number: & \\
\hline Article Type: & Full Paper \\
\hline Corresponding Author: & $\begin{array}{l}\text { Richard B. Jackman, PhD } \\
\text { University College London } \\
\text { London, London UNITED KINGDOM }\end{array}$ \\
\hline Corresponding Author E-Mail: & r.jackman@ucl.ac.uk \\
\hline Other Authors: & Maeve H. S. McLaughlin \\
\hline & Alexander C. Pakpour-Tabrizi, PhD \\
\hline Requested Editor: & Greg M Swain, Editor_Americas, Africa \\
\hline Keywords: & $\begin{array}{l}\text { Diamond } \\
\text { Electrochemical sensors } \\
\text { Nanoparticles } \\
\text { Polishing } \\
\text { Sensitivity }\end{array}$ \\
\hline Manuscript Classifications: & Carbon materials; Electrodes; Nanoelectrodes; Nanoparticles; Sensors \\
\hline Suggested Reviewers: & $\begin{array}{l}\text { Foord S. John } \\
\text { Professor, University of Oxford } \\
\text { john.foord@chem.ox.ac.uk } \\
\text { International expert }\end{array}$ \\
\hline & $\begin{array}{l}\text { Paul May } \\
\text { Professor, University of Bristol } \\
\text { paul.may@bristol.ac.uk } \\
\text { International expert }\end{array}$ \\
\hline Opposed Reviewers: & $\begin{array}{l}\text { Julie MacPherson } \\
\text { Professor, University of Warwick } \\
\text { J.MacPherson@warwick.ac.uk } \\
\text { Conflict of interest - we work with a sponsor the Warwick team wanted }\end{array}$ \\
\hline & $\begin{array}{l}\text { Mark Newton } \\
\text { Professor, University of Warwick } \\
\text { M.E.Newton@warwick.ac.uk } \\
\text { Conflict of interest we work with a sponsor that the Warwick team would have liked }\end{array}$ \\
\hline Abstract: & $\begin{array}{l}\text { This work compares polished and unpolished boron doped diamond (BDD) electrodes } \\
\text { decorated with two sizes of gold nanoparticles (AuNPs) for use as robust mercury } \\
\text { sensors in aquatic environments. The size of the catalytically active AuNPs on the } \\
\text { electrode surfaces was demonstrated to have a less significant effect on the sensitivity } \\
\text { for mercury detection than the surface preparation of the BDD. The lowest limits of } \\
\text { detection were achieved with the polished BDD electrodes, which both detected } \\
\text { mercury at a concentration of } 1 \text { pM, six orders of magnitude greater sensitivity than the } \\
\text { lowest detection limit of } 5 \mu \mathrm{M} \text { achieved with an unpolished BDD electrode, and high in } \\
\text { comparison with other reported electrode systems. }\end{array}$ \\
\hline Author Comments: & $\begin{array}{l}\text { We offer a powerful insight into how to make diamond electrodes perform better than } \\
\text { previously reported for the detection of mercury, a highly toxic metallic species in the } \\
\text { environment and often released, for example, by the activities of the oil and fracking } \\
\text { industries }\end{array}$ \\
\hline Section/Category: & Manuscripts from Europe (S. Campuzano Ruiz, J. Pingarrón) \\
\hline Additional Information: & \\
\hline Question & Response \\
\hline
\end{tabular}


Dedication

Submitted solely to this journal?

Yes

Has there been a previous version?

No

Powered by Editorial Manager ${ }^{\circledR}$ and ProduXion Manager ${ }^{\circledR}$ from Aries Systems Corporation 


\title{
Diamond electrodes for high sensitivity mercury detection in the aquatic environment: Influence of surface preparation and gold nanoparticle activity
}

\author{
Maeve H. S. McLaughlin, Alexander C. Pakpour-Tabrizi and Richard B. Jackman * \\ London Centre for Nanotechnology and Department of Electronic and Electrical Engineering, University College London, 17-19 \\ Gordon Street, London, WC1H 0AH, UK. \\ * e-mail: r.jackman@ucl.ac.uk
}

Received:

Accepted:

\begin{abstract}
This work compares polished and unpolished boron doped diamond (BDD) electrodes decorated with two sizes of gold nanoparticles (AuNPs) for use as robust mercury sensors in aquatic environments. The size of the catalytically active AuNPs on the electrode surfaces was demonstrated to have a less significant effect on the sensitivity for mercury detection than the surface preparation of the BDD. The lowest limits of detection were achieved with the polished BDD electrodes, which both detected mercury at a concentration of $1 \mathrm{pM}$, six orders of magnitude greater sensitivity than the lowest detection limit of 5 $\mu \mathrm{M}$ achieved with an unpolished BDD electrode, and high in comparison with other reported electrode systems.
\end{abstract}

Keywords: Diamond, Mercury, Gold, Nanoparticles

DOI: 10.1002/elan.

\section{Introduction}

Mercury is a highly toxic heavy metal that exists in concentrations up to $100 \mathrm{mg} / \mathrm{kg}$ within the Earth's mantle and may be released into the greater environment during the extraction and processing of crude oil [1]. Even in trace quantities mercury contamination poses a severe threat to human health because it tends to form complexes with ligands of biological matter, leading to an accumulation in the food chain [2]. The World Health Organisation guideline for the maximum safe quantity of mercury in potable water is $6 \mu \mathrm{gL}^{-1}$, ca. $30 \mathrm{nM}$ [3].

It is imperative that mercury can be detected in situ, where contamination may have occurred, rather than relying on post sample analysis in a remote laboratory. This is achievable through the use of an electrochemical detection procedure which can be built around portable equipment and rapidly depolyed and used in a wide range of locations, unlike the traditionally used optical methods such as inductively coupled plasma mass spectrometry or electrothermal atomic absorption spectrometry [4]. Moreover, with the emergence of the 'internet of things' there is significant interest in the use of networks of remote devices to give real-time monitoring over large environmental areas. To date, the published literature indicates that concentrations of mercury detected electrochemically can be down to levels of around $0.42 \mathrm{nM}$ using a glassy carbon electrode, modified with $36 \mathrm{~nm}$ diameter gold nanoparticles (AuNPs) [5]. The AuNPs act catalytically during the pre-concentration step of the square wave anodic stripping voltammetry (SWASV) measurements typically performed for electrochemical detection within an aqueous solution, which improves the sensitivity of the electrode.

Boron doped diamond (BDD) has been gaining interest as an electrode for use in the trace detection of analytes. In addition to the low background and capacitive currents associated with the material it has the widest 'electrochemical window' of any known electrode - that is the widest voltage range that can be applied without the onset of either oxidation or reduction of the solvent at the working electrode [6]. Therefore, a greater sensitivity for the detection of trace mercury in solution is expected with a BDD electrode decorated with AuNPs than with a glassy carbon electrode. The hardness and chemically inert nature of diamond also enables BDD electrodes to be used in environments where other electrodes would be damaged, which is particulary advantageous for the potential 
deployment of $\mathrm{Hg}$ sensors in the extreme condtions that can be encountered in various environmental locations.

Typical commercially available heavily boron doped diamond materials grown by chemical vapour deposition (CVD) methods are polycrystalline in nature; the presence of boron concentrations greater than $10^{20} \mathrm{~cm}^{-3}$ gives rise to quasi-metallic character, offering the conductivity required of the electrode [7]. The electronic properties of a BDD electrode are dependant on the boron concentration and graphitic carbon content of the diamond (which effects film morphology) $[7,8,9]$. Surface electronic properties are strongly influenced by the nature of the surface termination species present. The hydrogen rich CVD growth produces diamond which is naturally hydrogen terminated and therefore hydrophobic. H-terminated BDD is reported to have a slightly narrower electrochemical window than when oxygen terminated, however, as gold nanoparticles (AuNPs) show greater electrical contact to H-terminated BDD this has been used throughout this study $[9,10]$. Here, the influence of how the dispersion of AuNPs on the BDD surface affects the sensitivity of electrochemical measurements has been explored. This has been achieved through the novel approach of using a TEM grid to act as a shadow mask during gold deposition, producing a grid with $28 \mu \mathrm{m}$ square patches of AuNPs separated by $23 \mu \mathrm{m}$ gaps. Further, the influence of BDD surface roughness and NP size have been studied. Finally, information on the relative adhesion properties of different NPs on the differing surfaces has been assessed.

\section{Experimental methods}

10x10x0.5 mm, electrochemical grade BDD $\left([\mathrm{B}]>10^{20} \mathrm{~cm}^{-}\right.$ 3) substrates were purchased from Element Six Ltd (e6cvd.com). Initial experiments were performed with unpolished polycrystalline BDD, with a surface roughness $\mathrm{R}_{\mathrm{A}} \sim 50 \mu \mathrm{m}$. Further investigations used polished polycrystalline diamond (pBDD), where the surface roughness was reduced to $R_{A}$ values around $50 \mathrm{~nm}$. All chemicals unless otherwise stated were purchased from Sigma-Aldrich. Reverse osmosis derived water, resistivity $18 \mathrm{M} \Omega$, was used throughout.

\subsection{BDD surface preparation}

Prior to processing organic contaminants were removed from the BDD and pBDD surfaces with a 'Piranha' clean (3:1 v/v of $98 \% \mathrm{HCl}$ and $30 \% \mathrm{H}_{2} \mathrm{O}_{2}$ ) for 10 minutes [11]. Between experiments the electrodes were cleaned by application of 150 current pulses lasting $100 \mathrm{~ms}$, which alternated between $10 \mathrm{~mA} \mathrm{~cm}{ }^{-2}$ and $-10 \mathrm{~mA} \mathrm{~cm}^{-2}$, as has been previously shown to be effective [10].
The graphitic carbon content in the surface of each BDD and pBDD substrate was qualitatively assessed with a Renishaw micro-Raman spectrometer (532 nm laser source). The Raman analysis was performed with 20x magnification, $10 \mathrm{~s}$ exposure and an average was taken over ten accumulations, the microscope was calibrated using a silicon substrate. WiRE (v 2.0) software was used for data acquisition.

The BDD and pBDD substrates were hydrogen terminated in an AX5010 Seki Technotron Inc. reactor with H-plasma for 10 minutes at $700 \mathrm{C}$ platen temperature (Williamson Dual wavelength pyrometer), $800 \mathrm{~W}$ power, 40 Torr pressure. The hydrophilic-hydrophobic nature of the termination on the diamond surfaces was assessed with a Kruss DSA1 contact angle goniometer, using $4 \mu \mathrm{ml}$ water droplets. Kruss DSA1 v1.80 drop shape analysis software was used to determine the contact angle at the three-phase contact point between the water droplet and the electrode surface.

\subsection{AuNP deposition and stability}

A non-continuous $3 \mathrm{~nm}$ gold film was sputtered onto the BDD and pBDD electrodes with an Emscope SC500 gold sputter coater. The gold films were segregated into nanoparticles by an annealing process (often refered to as 'de-wetting', due to the role of surface tension) in a Solaris 150 Rapid Thermal Processing System under nitrogen at $400 \mathrm{C}$ for 5 minutes. To produce smaller AuNPs on some of the electrodes in a grid pattern the gold film was sputtered through a copper TEM grid (dimensions: $23 \mu \mathrm{m}$ bar, $28 \mu \mathrm{m}$ hole). This left 28 x $28 \mu \mathrm{m}$ squares of AuNPs separated by $23 \mu \mathrm{m}$ gaps on the BDD surface and influenced the size distribution of the NPs (as discussed later). An extended lifetime test consisting of ultrasonication in water for 10 minutes was performed on every electrode after the surface was coated with AuNPs. It will be seen (section 4.2) that changes provoked by the extended lifetime testing were modest but measurable. Since the electrode surfaces are likely to be more representative of an electrode under use in the field all subsequent sensing measurements were performed on electrodes following this sonication process.

\section{3. $\mathrm{Hg}^{2+}$ sensing}

SWASV measurements were used for $\mathrm{Hg}^{2+}$ detection, with a AuNP decorated BDD or pBDD working electrode, a $\mathrm{Ag} / \mathrm{AgCl} \mathrm{KCl}(3 \mathrm{M})$ reference electrode and a platinum counter electrode. A $0.1 \mathrm{M} \mathrm{HNO}_{3}$ electrolyte was used, being dosed with increasing concentrations of mercury 
nitrate from $1 \mathrm{pM}$ up to $1 \mathrm{mM} \mathrm{Hg}\left(\mathrm{NO}_{3}\right)_{2}$ to explore the lower detection limit available with each electrode. A bare BDD control electrode confirmed insufficient activity for effective sensing at any of the mercury concentrations measured here. $\mathrm{Hg}^{0}$ was pre-concentrated onto the working electrode surface by application of $0.35 \mathrm{~V}$ for 10 minutes [12]. A SWASV scan from 0.35 to $1.1 \mathrm{~V}$ with a $0.005 \mathrm{~V}$ potential step, $0.01 \mathrm{~V}$ amplitude and $10 \mathrm{~Hz}$ frequency was then applied [10], followed by the application of $150 \times 100 \mathrm{~ms}$ current pulses, alternating between $10 \mathrm{~mA} \mathrm{~cm}^{-2}$ and $-10 \mathrm{~mA} \mathrm{~cm}^{-2}$ to clean the working electrode surface [13].

\subsection{SEM}

A Zeiss XB1540 Crossbeam scanning electron microscope (10 kV operation voltage) was used to quantify the size and dispersion of AuNPs on the electrode surfaces. Scans from five random locations across each substrate were analysed with ImageJ software to calculate the average size and surface coverage of AuNPs on each electrode.

\section{Results}

\subsection{BDD characterisation}

The graphitic carbon content on the surface of the BDD and pBDD substrates was qualitativley assessed with Raman spectroscopy (Figure 1).

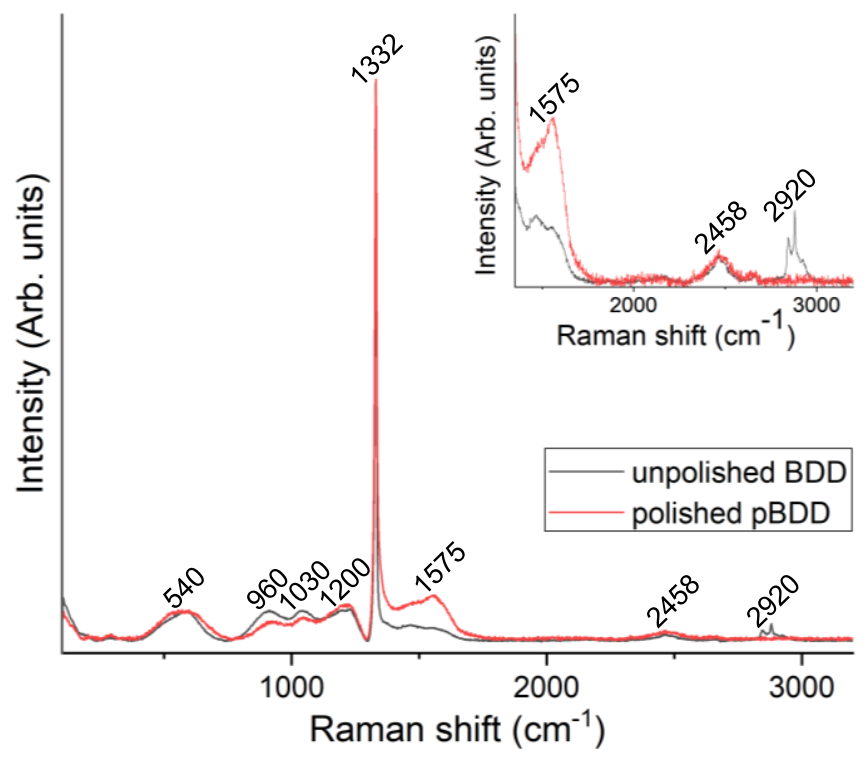

Fig. 1. Raman spectrum of unpolished BDD substrate revealing the distinctive $1332 \mathrm{~cm}^{-1}$ peak of the diamond carbon phase, and (inset) second order Raman spectrum (black) and similar measurements for the polished pBDD substrate materials (red).
The degree of the hydrogen termination achieved following processing of each electrode was explored using contact angle measurements, which gave an insight into the level of hydrophobicity imparted by the treatment (Table 1).

Table 1. Three-phase contact angle on each substrate surface from contact angle measurements with $4 \mu \mathrm{ml}$ water droplets.

\begin{tabular}{lll}
\hline Electrode & Contact angle & Error \\
\hline BDD + 10 nm AuNPs & 108.5 & \pm 0.41 \\
BDD + 22 nm AuNPs & 107.3 & \pm 0.42 \\
pBDD + 13 nm AuNPs & 115.3 & \pm 0.19 \\
pBDD + 23 nm AuNPs & 113.1 & \pm 1.01 \\
\hline
\end{tabular}

\subsection{AuNP production and stability}

The AuNPs produced from gold films that were sputtered onto the electrode surface through a copper TEM grid (dimensions: $23 \mu \mathrm{m}$ bar, $28 \mu \mathrm{m}$ hole) resulted in smaller AuNPs inside the gaps left by the TEM grid than those produced on the same electrode outside the area covered by the TEM grid (Figure 2).

(a)

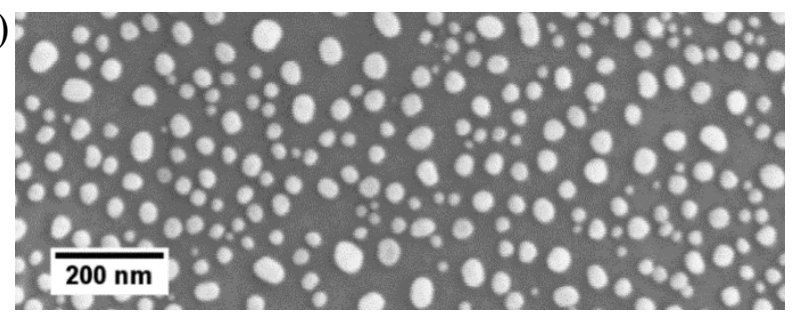

(b)

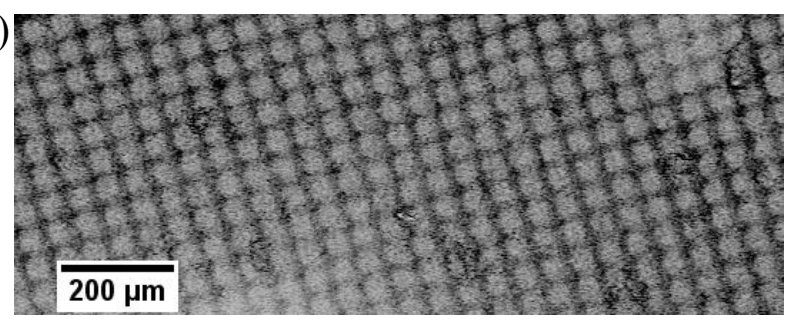

(c)

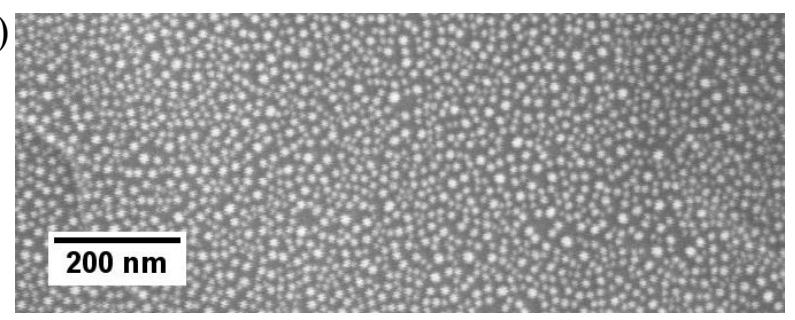

Fig. 2. (a) Average diameter $23 \mathrm{~nm}$ AuNPs on pBDD electrode. (b) Patterned gold on pBDD surface after TEM grid was used as a shadow mask. (c) Average diameter $13 \mathrm{~nm}$ AuNPs on pBDD electrode, inside the holes of the TEM grid shadow mask. 
Five SEM scans from random locations across each substrate were analysed using the 'analyse particles' function in ImageJ software to calculate the average AuNP diameter and density of coverage on each substrate and the standard deviation of this number. The physical stability of AuNPs on the electrodes was tested by comparing each surface with SEM before and after an accelerated lifetime test which involved ultrasonication in an aqueous medium for 10 minutes; the results are shown in Figure 3 for AuNPs formed by the various procedures described above.

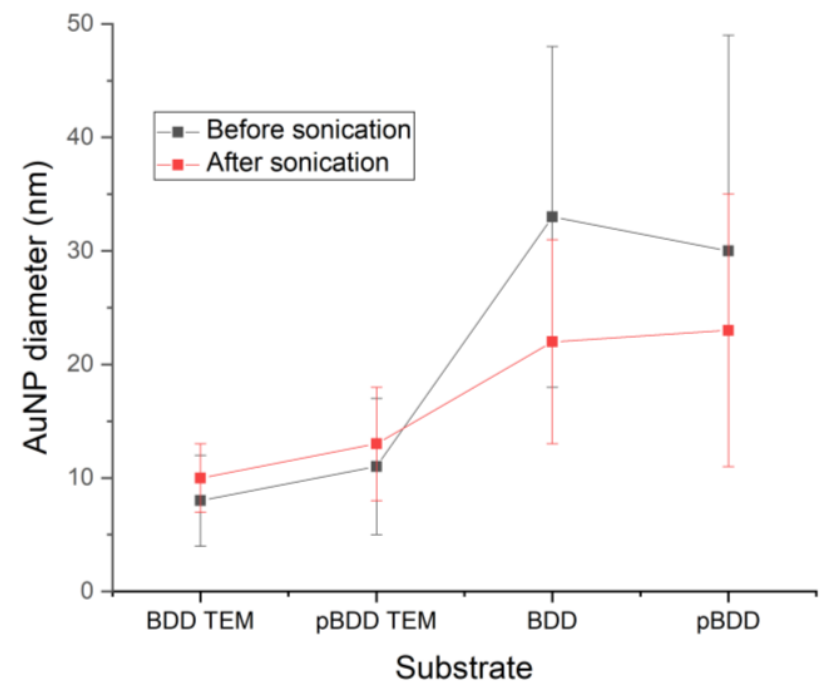

Fig. 3. Comparison of AuNP average diameter and standard deviation on each electrode before and after ultrasonication in water (10 minutes), the AuNPs on the first two substrates were deposited through a TEM grid (copper, $23 \mu \mathrm{m}$ bar, $28 \mu \mathrm{m}$ hole).

The chemical stability of the AuNPs on each electrode was determined by analysing SEM scans taken on five random areas across each electrode before and after the electrodes were used for SWASV measurements. An average AuNP diameter and the standard deviation of that value was calculated for each electrode before and after the SWASV measurements by again using the 'analyse particles' function of ImageJ software; the results are shown in Figure 4. The SEM images themselves are shown in figure 5 where the persistence of the AuNPs can be clearly seen.

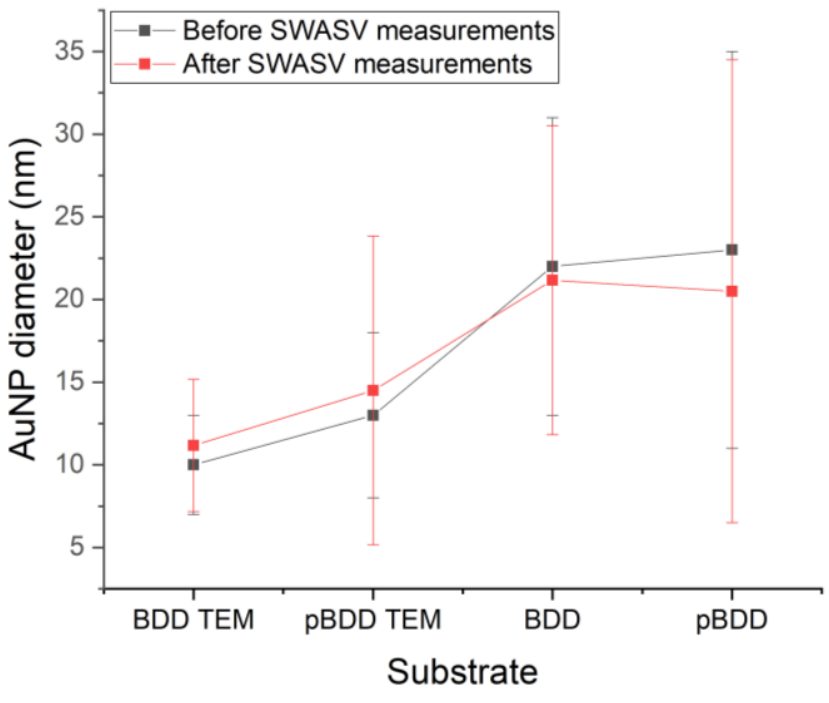

Fig. 4. Comparison of AuNP average diameter and standard deviation on each electrode before and after SWASV measurements, the AuNPs on the first two substrates were deposited through a TEM grid (copper, $23 \mu \mathrm{m}$ bar, $28 \mu \mathrm{m}$ hole).

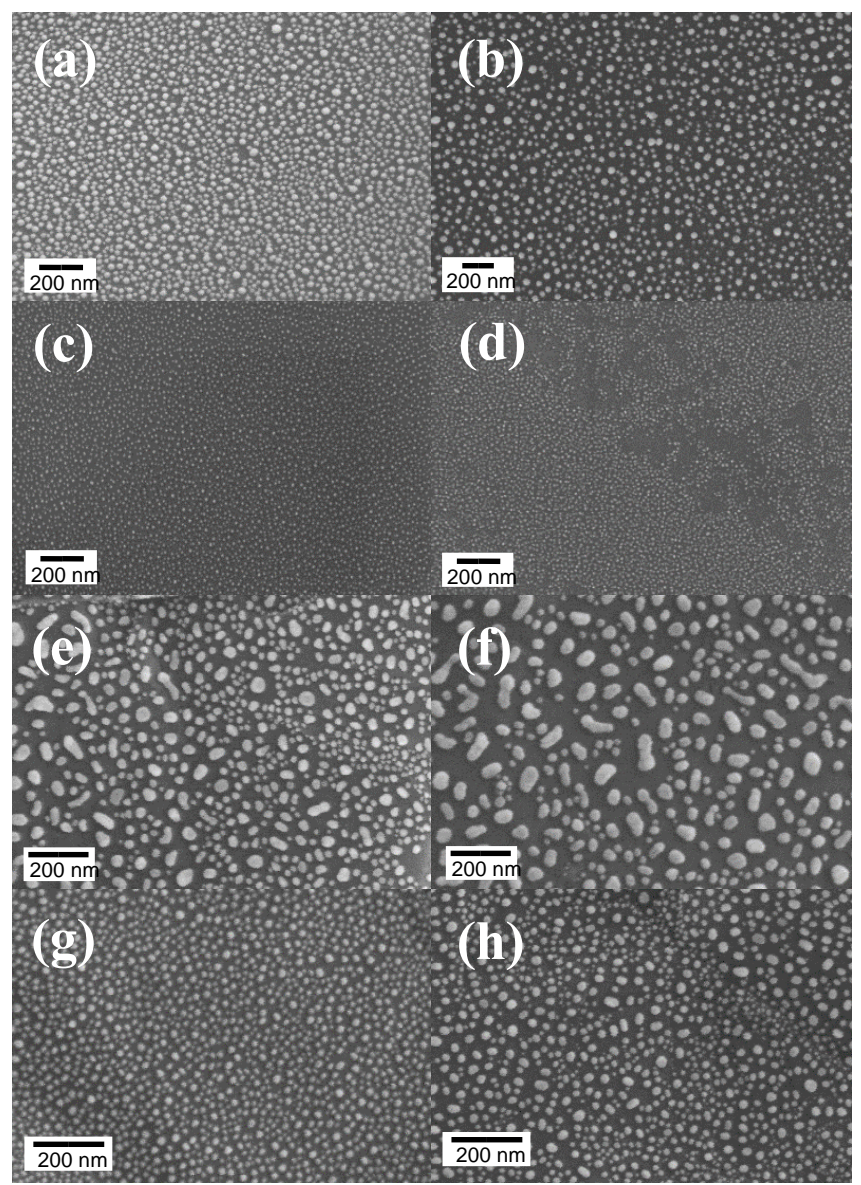

Fig. 5. SEM scans of (a) BDD electrode decorated with $22 \mathrm{~nm}$ AuNPs before SWASV measurements and (b) afterwards, BDD electrode decorated with $10 \mathrm{~nm}$ AuNPs before (c) and after (d) SWASV measurements, pBDD electrode decorated with $23 \mathrm{~nm}$ AuNPs before (e) and after (f) SWASV measurements and pBDD electrode decorated with $13 \mathrm{~nm}$ AuNPs before (g) and after (h) SWASV measurements. 


\subsection{Electrochemical measurements}

\section{(a) Mercury concentration $1 \mathrm{pM}$}

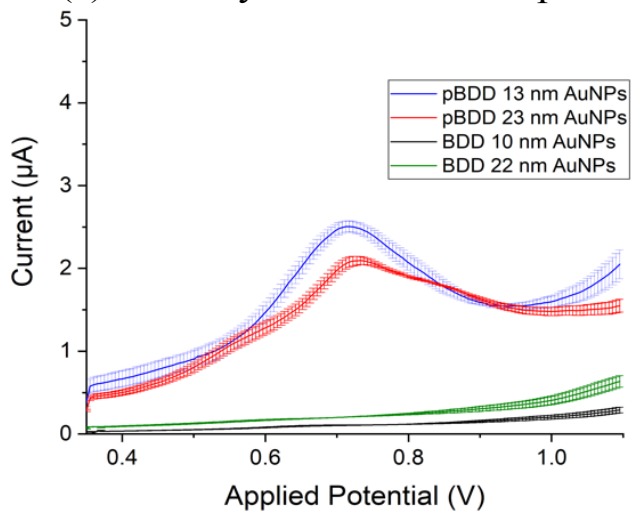

(b) Mercury concentration $5 \mu \mathrm{M}$

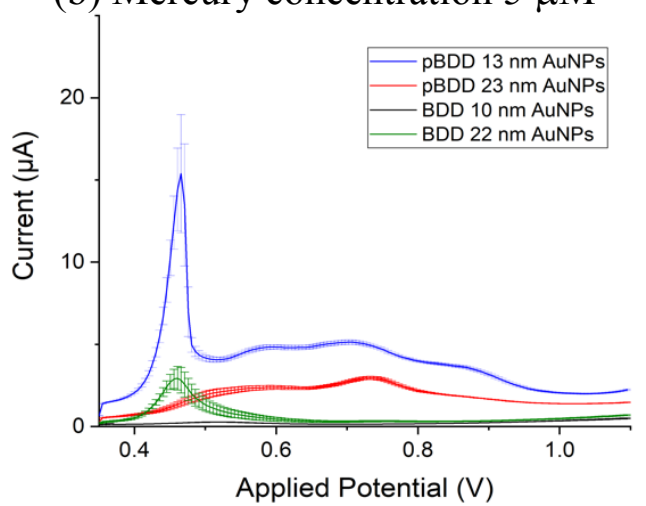

(c) Mercury concentration $0.1 \mathrm{mM}$

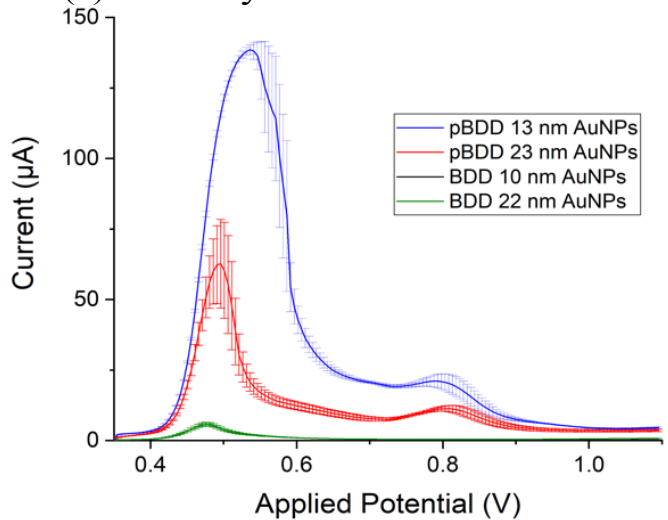

(d) Mercury concentration $1 \mathrm{mM}$

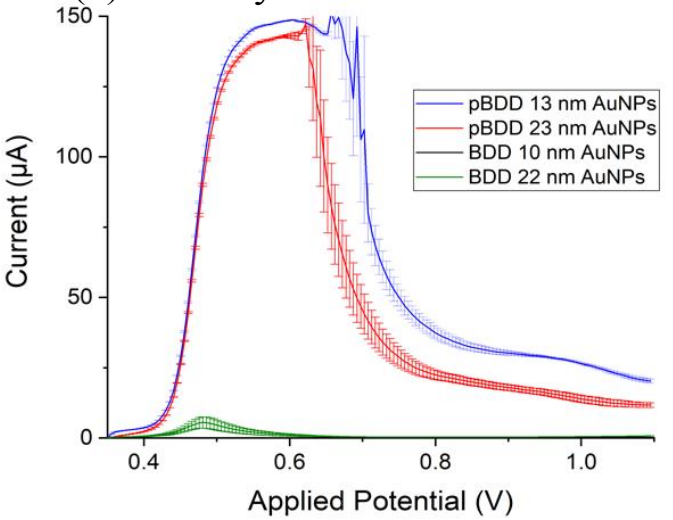

Fig. 6. Comparison of SWASV average scans and standard error with $0.005 \mathrm{~V}$ potential step, $0.01 \mathrm{~V}$ amplitude, $10 \mathrm{~Hz}$ frequency for each electrode at $\mathrm{Hg}^{2+}$ concentrations (a) $1 \mathrm{pM}$, (b) $5 \mu \mathrm{M}$, (c) $0.1 \mathrm{mM}$ and (d) $1 \mathrm{mM}$ (other SWASV scans for all concentrations measured within this range are available within the supporting information).

The SWASV measurements made with each electrode were repeated four times at each concentration of mercury tested. The average observed current of the four repeats for each electrode at a range of mercury concentrations is plotted in Figure 6 with the standard error for each value. To test whether the sensitivity for mercury detection is affected by the diffusion of ions to the electrode surface the SWASV measurements for the BDD electrode decorated with $22 \mathrm{~nm}$ AuNPs were repeated at a lower frequency of $0.5 \mathrm{~Hz}$ and therefore a 20x slower scan speed of $0.0025 \mathrm{Vs}^{-1}$ (Figure 7).

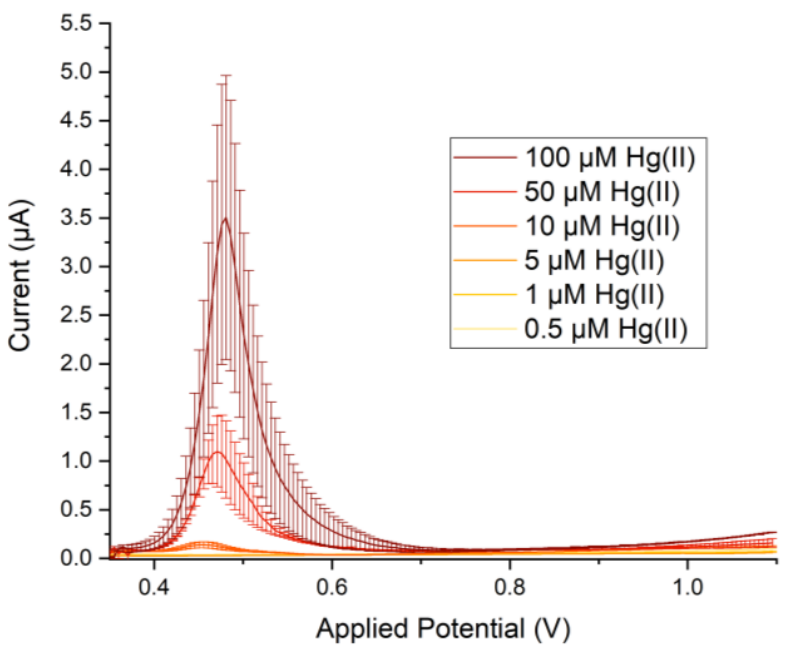

Fig. 7. Average SWASV measurements taken from four repeats and the standard error of the observed current when voltage of $0.005 \mathrm{~V}$ potential step, $0.01 \mathrm{~V}$ amplitude, $0.5 \mathrm{~Hz}$ frequency was applied for each concentration of mercury in electrolyte from 0.5 $-100 \mu \mathrm{M}$.

The maximum current peak observed for the reoxidation of mercury from $\mathrm{Hg}^{0}$ to $\mathrm{Hg}^{2+}$ at each detectable concentration was plotted against the mercury concentration in the electrolyte during that measurement for the BDD electrode decorated with $22 \mathrm{~nm}$ AuNPs during SWASV scans at both $10 \mathrm{~Hz}$ and $0.5 \mathrm{~Hz}$ frequency (Figure 8).
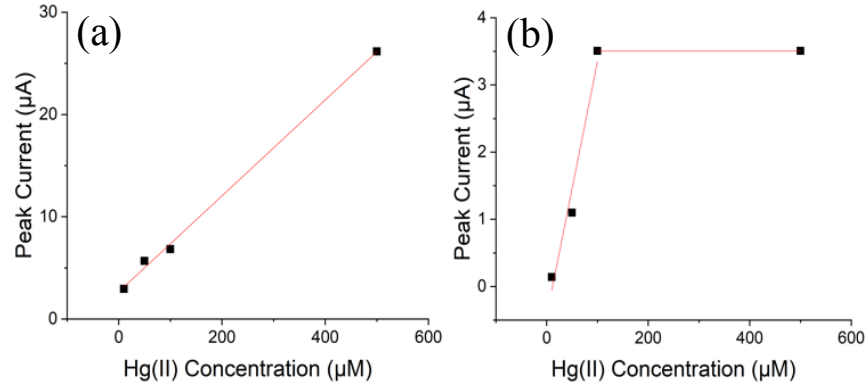
Fig. 8. (a) Peak current peak for each concentration of $\mathrm{Hg}$ (II) in solution detected with BDD electrode decorated with $22 \mathrm{~nm}$ AuNPs upon application of SWASV scan with $0.005 \mathrm{~V}$ potential step, $0.01 \mathrm{~V}$ amplitude, $10 \mathrm{~Hz}$ frequency and (b) with $0.5 \mathrm{~Hz}$ frequency.

\section{Discussion}

\subsection{Electrode characterisation}

The graphitic carbon content on the surface of each electrode was determined with Raman spectroscopy. The shape of the characteristic $1332 \mathrm{~cm}^{-1}$ diamond carbon peak is indicative of the crystalline quality of the substrate, as a lower quality diamond that contains more defects will have a shorter phonon lifetime and broader line width than seen in Figure 1 [6]. The size of the non-diamond carbon $\mathrm{G}$ peak at $1575 \mathrm{~cm}^{-1}$ in relation to the characteristic $\mathrm{sp}^{3}$ diamond carbon peak at $1332 \mathrm{~cm}^{-1}$ can be used to qualitatively assess the proportion of non-diamond carbon within the diamond surface structure [14]. In Figure 1 the intensity of the $1575 \mathrm{~cm}^{-1}$ peak is significantly smaller than the $\mathrm{sp}^{3}$ diamond carbon peak at $1332 \mathrm{~cm}^{-1}$ for both BDD and $\mathrm{pBDD}$, it is therefore considered that the proportion of non-diamond carbon in the substrates is very low. This is confirmed by the presence of the second order $\mathrm{sp}^{2}$ peak at $2920 \mathrm{~cm}^{-1}$, which is also of a significantly smaller magnitude than the $1332 \mathrm{~cm}^{-1}$ peak. A higher proportion of non-diamond carbon on the pBDD surface, as a result of polishing damage, is indicated in Figure 1, due to the absence of the second order $\mathrm{sp}^{2}$ peak at $2920 \mathrm{~cm}^{-1}$. In addition, there is a smaller ratio (16:1) between the 1332 $\mathrm{cm}^{-1}$ and $1575 \mathrm{~cm}^{-1}$ peaks in Figure 1 than the ratio between the same peaks for the BDD substrate in Figure 1 (47:1). Increasing the proportion of $\mathrm{sp}^{2}$ carbon in a BDD electrode has been shown to favour electrochemical oxidation and have a significant effect on the electrode reaction kinetics of some redox systems $[15,16]$.

The broad peaks between $500 \mathrm{~cm}^{-1}$ and $1200 \mathrm{~cm}^{-1}$ in the Raman spectra for both BDD and pBDD indicate a high boron doping concentration, greater than $10^{20}$ boron atoms $\mathrm{cm}^{-2}$ within the diamond structures (Figure 1) [17]. The high boron concentration is further confirmed by the asymmetry at the base of the $1332 \mathrm{~cm}^{-1}$ peak - known as the Fano resonance, which corresponds to the onset of metal-like conductivity in the diamond as a result of the boron impurity band transitioning into a continuum state [18].

The average surface termination of the electrodes following the AuNP deposition was assessed via contact angle measurements (Table 1). The contact angle for a 4 $\mu \mathrm{L}$ water droplet on each electrode was greater that $90^{\circ}$, meaning that all of the electrodes were hydrophobic and therefore predominantly hydrogen terminated [19]. It is established in existing literature that the hydrophobic nature of heavily doped BDD results in water contact angles $>105^{\circ}[20]$. The results shown in Table 1 reflect that the hydrogen termination of the electrodes used here was preserved during the AuNP deposition process, which was ensured by annealing the gold films in an inert atmosphere during the de-wetting stage. There is evidence that the contact angle of a surface is reduced when the roughness of that surface is increased, which is why the BDD electrodes had smaller contact angles than the pBDD electrodes [21].

\subsection{AuNP characterisation}

The AuNP diameter and surface coverage on each electrode was calculated from an average of five SEM scans taken across random areas on each electrode surface. The two electrodes where the gold was deposited onto the surface through a copper TEM grid (dimensions: $23 \mu \mathrm{m}$ bar, $28 \mu \mathrm{m}$ hole) which acted as a shadow mask, resulted in AuNPs with $>10 \mathrm{~nm}$ smaller diameter. During the gold deposition in the Emscope SC500 the substrate holder is held at a positive potential, which induces a charge in both the BDD and the TEM grid placed on its surface. The positively charged copper TEM grid has a filtering effect on the gold, leading to smaller AuNPs inside the holes of the grid, most likely due to its electrostatic field affecting the sputtered gold ions in the chamber [22].

The SEM scans used to determine the average AuNP diameter on each electrode were compared to SEM scans taken following an extended lifetime test of ultrasonication in water for 10 minutes with each AuNP decorated electrode (Figure 3 and supporting information). As seen in Figure 3 the ultrasonication of the electrodes resulted in a smaller standard deviation in AuNP diameter for every electrode. Where the gold was sputtered through a TEM grid following the ultrasonication of the electrode the AuNP average diameter increased, due to the agglomeration of a proportion of the smallest nanoparticles. Whereas, when the gold was sputtered onto the electrodes without a TEM grid shadow mask the AuNP average diameter decreased after sonication, as during this process a proportion of the larger AuNPs were partially broken down into a mixture of nanoparticle diameters. This discrepancy can be explained by the filtering effect of the TEM grid on the gold during sputtering, which affected the bonding energy between the diamond surface and the AuNPs in comparison to the larger AuNPs produced from gold sputtered onto the electrodes in the absence of a TEM grid shadow mask. The weaker adhesion of AuNPs to the 
BDD surfaces when the gold was deposited through a TEM grid is demonstrated by the loss of some AuNPs during SWASV measurements using the BDD electrode decorated with $10 \mathrm{~nm}$ AuNPs, depicted in the SEM images of Figure $5 \mathrm{c}$ and $5 \mathrm{~d}$.

The chemical stability of the AuNPs on the BDD electrodes was evaluated by comparison of the SEM scans taken prior to the SWASV measurements with an average of five SEM scans taken across random areas on each electrode after the mercury detection measurements. The AuNPs on each electrode had a smaller average change in diameter following the SWASV measurements in comparison to the diameter change observed following the extended lifetime testing (Figure 3). However, the standard deviation in AuNP diameter increased during the SWASV measurements on every electrode due to agglomeration of some of the smallest nanoparticles with the adjacent AuNPs of largest diameter. This produced larger maximum diameter AuNPs and reduced the overall surface coverage of the nanoparticles on the electrode surfaces. However, the majority of the AuNPs were unchanged, resulting in only small changes in the average AuNP diameter on each electrode. A greater increase in the standard deviation of the AuNP average diameter was observed on the pBDD electrodes than the unpolished $\mathrm{BDD}$ as on the polished electrodes there was lower adhesion and a greater proportion of the AuNPs agglomerated, resulting in a lower coverage of nanoparticles after the SWASV measurements (Figure 5).

\section{3. $\mathrm{Hg}^{2+}$ characterisation}

Excluding the measurements made with the BDD electrode decorated with $22 \mathrm{~nm}$ AuNPs taken with a frequency of $0.5 \mathrm{~Hz}$ (Figure 7) all of the SWASV measurements used for $\mathrm{Hg}(\mathrm{II})$ detection were performed at a scan rate $0.05 \mathrm{Vs}^{-1}$ (Figure 6 and supporting information). The reoxidation of $\mathrm{Hg}^{0}$ at the working electrode surface to $\mathrm{Hg}(\mathrm{II})$ during the SWASV measurements is observed at $0.5 \mathrm{~V}$. At higher mercury(II) concentrations $(>10 \mu \mathrm{M})$ a competing oxidation reaction of the formation of a mercuric dimer was observed at 0.8$0.9 \mathrm{~V}$ with every electrode [10].

The pBDD electrodes had greater sensitivity and therefore detected lower concentrations of mercury(II) in the electrolyte than the unpolished BDD electrodes. The polishing process is known to encourage defect sites to propogate through the diamond lattice to a depth of several microns and creates surface-stable $\mathrm{sp}^{2}$ carbon [23]. These effects in addition to the size and distribution of the AuNPs on the pBDD electrode surface can explain the superior sensitivity achieved with these electrodes.
The pBDD electrode decorated with $13 \mathrm{~nm}$ AuNPs showed greater sensitivity (higher observed current) than the pBDD electrode decorated with $23 \mathrm{~nm}$ AuNPs, due to the increased surface area of the catalytically active AuNPs. However, the same trend was not observed with the unpolished BDD electrodes for which the electrode with larger AuNPs had a lower detection limit for mercury. This is because a proportion of the average $10 \mathrm{~nm}$ diameter AuNPs on the BDD electrode were dislodged from the electrode surface during the SWASV measurements (Figure 5d), resulting in a reduction of the area coated with catalytically active AuNPs. A lower adhesion of AuNPs on the diamond surface which were produced by annealing gold films deposited through a TEM grid was observed on both the polished and unpolished diamond electrodes. This is due to the electrostatic effect of the TEM grid during the gold deposition, throughout which a bias was applied to the substrate and therefore TEM grid placed on the diamond, causing them to become positively charged. This resulted in smaller average diameter AuNPs inside the gaps left by the TEM grid, with reduced adhesion to the diamond surface in comparison to the AuNPs outside the TEM grid shadow mask.

Significantly higher sensitivity was achieved with the polished diamond substrates (50 $\mathrm{nm}$ surface roughness) than the unpolished BDD substrates $(50 \mu \mathrm{m}$ surface roughness) regardless of the AuNP diameter on the electrode surface. Both pBDD electrodes detected $1 \mathrm{pM}$ concentrations of mercury, whereas the first detectable concentration with a BDD electrode was $5 \mu \mathrm{M}$, with the BDD electrode decorated with $22 \mathrm{~nm}$ AuNPs. The sensitivity performance of the pBDD electrodes is excellent when compared to recent reports of alternative electrode materials for this purpose which are in the $\mu \mathrm{M}$ nM range [24,25].

Mercury detection at the BDD electrodes was shown to be unaffected by the diffusion of ions to the electrode surface because when the SWASV measurements using the BDD electrode decorated with $22 \mathrm{~nm}$ AuNPs were repeated at a 20x slower scan speed the lower limit of detection was the same as for the previous SWASV measurements $(5 \mu \mathrm{M})$. A linear relationship between the peak observed current when mercury was detected and the concentration of mercury(II) in the electrolyte for that SWASV measurement was observed for the BDD electrode decorated with $22 \mathrm{~nm}$ AuNPs at $10 \mathrm{~Hz}$ scan frequency (Figure 8a). Although the same linear electrode response was also initially observed for the SWASV measurements at the lower scan frequency of $0.5 \mathrm{~Hz}$, the electrode became saturated at $100 \mu \mathrm{M}$ mercury concentration. The saturation of the electrode resulted in no change in the maximum current of the mercury 
oxidation peak at concentrations greater than $100 \mu \mathrm{M}$ (Figure $8 \mathrm{~b}$ ). This demonstrates that the BDD electrode becomes saturated at high concentrations of mercury when slow SWASV scan speeds are used. However, as the same sensitivity of $5 \mu \mathrm{M}$ was recorded using the BDD electrode decorated with $22 \mathrm{~nm}$ AuNPs at both scan speeds this highlights that it is not necessary to reach saturation of the sensor to achieve repeatable measurements.

\section{Conclusions}

This work presented has been an investigation as to how the dispersion of AuNPs on the BDD electrode surfaces and the roughness of the BDD surface affects the sensitivity of electrochemical measurements for mercury detection in aqueous solution. Although greater sensitivity was achieved with the pBDD electrode decorated with 13 $\mathrm{nm}$ AuNPs than the pBDD electrode decorated with $23 \mathrm{~nm}$ AuNPs overall the size of the catalytically active AuNPs on the electrode surfaces was demonstrated to have a smaller effect on the sensitivity for mercury detection than the surface preparation of the BDD surface.

A lower limit of detection was achieved with both polished BDD electrodes than with either unpolished BDD electrode. This was proven to not be caused by the diffusion of ions to the electrode surface through repetition of the SWASV measurements with the BDD electrode decorated with $22 \mathrm{~nm}$ AuNPs at a 20x slower scan speed. This resulted in the same sensitivity as during the SWASV measurements using the same electrode at the higher scan rate, which demonstrates that the BDD electrodes are well suited to rapid in situ measurements for mercury detection in aqueous environments.

The superior sensitivity of the pBDD electrodes can be explained by the higher graphitic carbon content on these electrodes, which was caused by polishing damage and characterised with Raman spectroscopy. The extreme sensitivity, pM, displayed by the pBDD electrodes, allied to the demonstrated tenacious nature of the attached AuNPs during repeated measurement cycles and accelerated lifetime (ultrasonic) tests suggests a promising future for this approach for the realisation of robust trace $\mathrm{Hg}$ detectors for a wide range of aqueous environments.

\section{Acknowledgements}

One of us (MHSM) thanks the UKs Engineering and Physical Sciences Research Council (EPSRC) and Schlumberger Cambridge Research Ltd for the award of a CASE PhD studentship (EP/N509577/1). We gratefully acknowledge Débora Campos de Faria of Schlumberger
Cambridge Research Ltd for useful discussions and feedback on this manuscript.

\section{References}

[1] J. H. Duffus 1980 Edward Arnold Publishers Ltd. Environmental Toxicology

[2] G. Aragay, J. Pons, A. Merkoçi Chemical Reviews 2011, 111, 3433-3458

[3] D. Lang 2017 World Health Organization Guidelines for Drinking-water Quality

[4] F. Arduini, J. Q. Calvo, G. Palleschi, D. Moscone, A. Amine Trends in Analytical Chemistry 2010, 29, 1295-1304

[5] T. Hezard, K. Fajerwerg, D. Evrard, V. Collière, P. Behra, P. Gros Journal of Electroanalytical Chemistry 2012, 664, 4652

[6] J. V. Macpherson Physical Chemistry Chemical Physics 2015, 17, 2935-2949

[7] A. Vanhove, J. de Sanoit, M. Mailley, A. Pinault, F. Jomard, P. Bergonzo Physica Status Solidi (A) 2009, 206, 2063-2069

[8] T. Watanabe, Y. Honda, K. Kanda, Y. Einaga Pysica Status Solidi (A) 2014, 211, 2709-2717

[9] D. Tryk, K. Tsunozaki, T. N. Rao, A. Fujishima Diamond and Related Materials 2001, 10, 1804-1809

[10] J. Svanberg-Larsson, G. W. Nelson, S. E. Steinvall, B. F. Leo, E. Brooke, D. J. Payne, J. S. Foord Electroanalysis 2016, 28, 88-95

[11] C. V. Nguyen, R. Bartali, L. Crema, G. Speranza Colloids and Surfaces A: Physiochemical and Engineering Aspects 2016, 498, 231-238

[12] L. Laffont, T. Hezard, P. Gros, L. E. Heimbürger, J. E. Sonke, P. Behra, D. Evrard Talanta 2015, 141, 26-32

[13] R. Kiran, E. Scorscone, J. de Sanoit, J. C. Arnault, P. Mailey, P. Bergonzo Journal of the Electrochemical Society 2013, 160, H67-H73

[14] A. C. Ferrari, J. Robertson Philosphical Transactions of the Royal Society A: Mathematical, Physical and Engineering Sciences 2004, 362, 2477-2512

[15] D. M. de Araújo, P. Cañizares, C. A. Martínez-Huitle, M. A. Rodrigo Electrochemistry Communications 2014, 47, $37-40$

[16] J. A. Bennett, J. Wang, Y. Show, G. M. Swain Journal of the Electrochemical Society 2004, 151, E306-E313

[17] S. Prawer, R. J. Nemanich Philosphical Transactions of the Royal Society A: Mathematical, Physical and Engineering Sciences 2004, 362, 2537-2565

[18] J. W. Ager, W. Walukiewicz, M. McCluskey, M. A. Plano, M. I. Landstrass Applied Physics Letters 1995, 66, 616-618

[19] K. Y. Law The Journal of Physical Chemistry Letters 2014, 5, 686-688

[20] A. F. Azevedo, M. R. Baldan, N. G. Ferreira Journal of Physics and Chemistry of Solids 2013, 74, 599-604

[21] E. Bormashenko Low Temperature Physics 2016, 42, 622-635

[22] T. A. Thorson, R. D. Durst, R. J. Fonck, L. P. Wainwright Physics of Plasmas 1997, 4, 4-15 


\section{Full Paper}

[23] T. Schuelke, T. A. Grotjohn Diamond and Related Materials 2013, 32, 17-26

[24] E. Eksin, A. Erdem, T. Fafal, B. Kivçak Electroanalysis 2019, 31, 1
ELECTROANALYSIS

[25] H. L. Nguyen, H. H. Cao, D. T. Nguyen, V. A. Nguyen Electroanalysis 2016, 29, 595-601 
Click here to access/download Additional Material - Author Graphical Abstract.tif 
Click here to access/download Supporting Information

Supporting information-Electroanalysis template.docx 